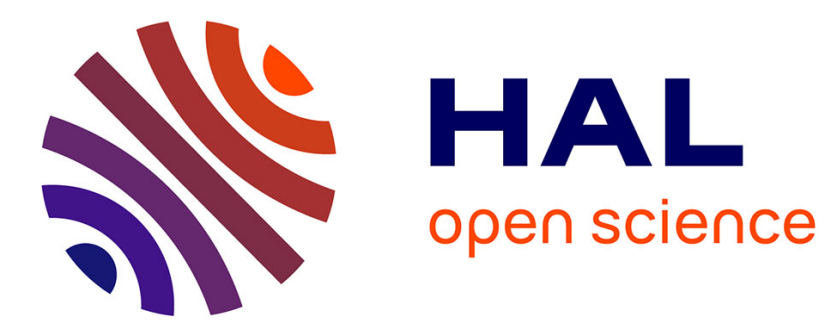

\title{
Synchrotron X-ray diffraction experiments with a prototype hybrid pixel detector
}

Christophe Le Bourlot, P Landois, S Djaziri, P.-O Renault, Eric Le Bourhis, P. Goudeau, M Pinault, M. Mayne-L'Hermite, B. Bacroix, D Faurie, et al.

\section{To cite this version:}

Christophe Le Bourlot, P Landois, S Djaziri, P.-O Renault, Eric Le Bourhis, et al.. Synchrotron X-ray diffraction experiments with a prototype hybrid pixel detector. Journal of Applied Crystallography, 2012, 45, pp.38-47. 10.1107/S0021889811049107 . hal-01203672

\section{HAL Id: hal-01203672 \\ https://hal.science/hal-01203672}

Submitted on 23 Sep 2015

HAL is a multi-disciplinary open access archive for the deposit and dissemination of scientific research documents, whether they are published or not. The documents may come from teaching and research institutions in France or abroad, or from public or private research centers.
L'archive ouverte pluridisciplinaire HAL, est destinée au dépôt et à la diffusion de documents scientifiques de niveau recherche, publiés ou non, émanant des établissements d'enseignement et de recherche français ou étrangers, des laboratoires publics ou privés. 


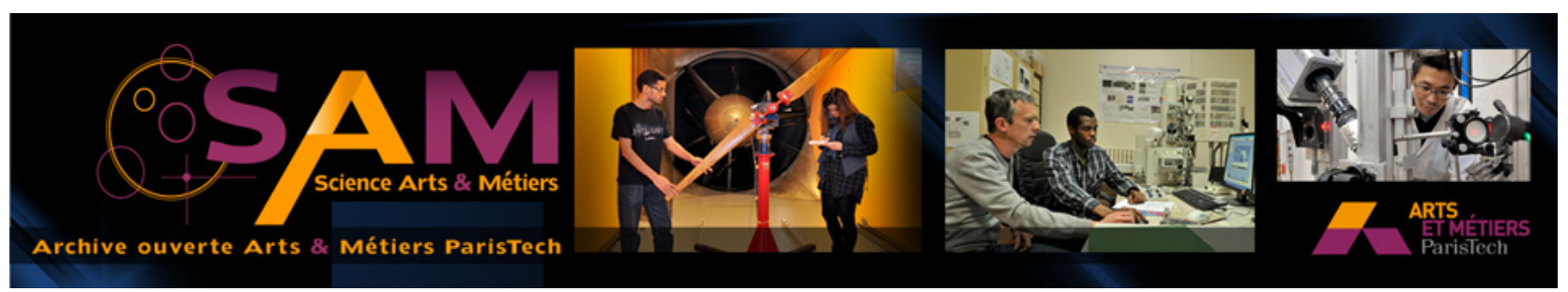

\section{Science Arts \& Métiers (SAM)}

is an open access repository that collects the work of Arts et Métiers ParisTech researchers and makes it freely available over the web where possible.

This is an author-deposited version published in: http://sam.ensam.eu

Handle ID: .http://hdl.handle.net/10985/10146

\section{To cite this version :}

C LE BOURLOT, P LANDOIS, S DJAZIRI, P.-O RENAULT, E LE BOURHIS, P GOUDEAU, M MAYNE-L'HERMITE, B. BACROIX, M PINAULT, D FAURIE, O CASTELNAU, P LAUNOIS, S ROUZIERE - Synchrotron X-ray diffraction experiments with a prototype hybrid pixel detector Journal of Applied Crystallography - Vol. 45, p.38-47 - 2012 


\title{
Synchrotron X-ray diffraction experiments with a prototype hybrid pixel detector
}

\author{
C. Le Bourlot, ${ }^{\text {a,d }}$ P. Landois, ${ }^{\mathrm{b}}$ S. Djaziri, ${ }^{\mathrm{c}}$ P.-O. Renault, ${ }^{\mathrm{c}}$ E. Le Bourhis, ${ }^{\mathrm{c}}{ }^{\mathrm{P}}$. \\ Goudeau, ${ }^{\mathrm{c}}$ M. Pinault, ${ }^{\mathrm{e}}$ M. Mayne-L'Hermite, ${ }^{\mathrm{e}}$ B. Bacroix, ${ }^{\mathrm{a}}$ D. Faurie, ${ }^{\mathrm{a}} \mathrm{O}$. \\ Castelnau, $^{d}$ P. Launois ${ }^{b}$ and $S$. Rouzière ${ }^{b *}$ \\ aLSPM, CNRS, Institut Galilée, Université Paris 13, Avenue J. Clément, 93430 Villetaneuse, France, \\ bLPS, Université Paris-Sud, CNRS, UMR8502, 91405 Orsay Cedex, France, 'Institut Pprime,

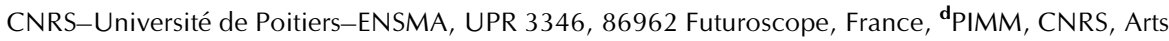 \\ et Métiers ParisTech, 151 Boulevard de l'Hôpital, 75013 Paris, France, and 'éF, CEA CNRS URA \\ 2453, CEA Saclay, 91191 Gif-sur-Yvette Cedex, France. Correspondence e-mail: \\ stephan.rouziere@u-psud.fr
}

\begin{abstract}
A prototype X-ray pixel area detector (XPAD3.1) has been used for X-ray diffraction experiments with synchrotron radiation. The characteristics of this detector are very attractive in terms of fast readout time, high dynamic range and high signal-to-noise ratio. The prototype XPAD3.1 enabled various diffraction experiments to be performed at different energies, sample-todetector distances and detector angles with respect to the direct beam, yet it was necessary to perform corrections on the diffraction images according to the type of experiment. This paper is focused on calibration and correction procedures to obtain high-quality scientific results specifically developed in the context of three different experiments, namely mechanical characterization of nanostructured multilayers, elastic-plastic deformation of duplex steel and growth of carbon nanotubes.
\end{abstract}

\section{Introduction}

A new generation of X-ray detectors has recently appeared in the wake of third-generation synchrotron facilities. The currently available bright synchrotron sources deliver high $\mathrm{X}$-ray photon flux, allowing experimenters to perform timeresolved measurements while detecting very weak signals. Such experiments require the use of specific detectors, enabling high counting rates with a large dynamic range, low electronic noise and short acquisition time. Hybrid pixel array detectors based on single-photon-counting processes have been developed over the past few years, for example MEDIPIX (Ponchut et al., 2007), XPAD (Basolo et al., 2008), PILATUS (Henrich et al., 2009) and MEDIPIX/TIMEPIX (Teyssier et al., 2011); these instruments demonstrate intrinsically low readout noise, high signal-to-noise ratio, fast readout time (down to a few milliseconds), high dynamic range and linearity limit, high framing rate, and the possibility to suppress fluorescence background by setting an energy threshold individually for each pixel. Moreover, hybrid pixel detectors can be designed with a large detection surface, giving access to a wide range of two-dimensional diffraction techniques.

In this work, we shall focus on the conditions of use of the XPAD3.1 hybrid pixel detector (Medjoubi et al., 2010), in the early phases of development of the prototype available at the synchrotron facilities SOLEIL and the CRG-D2AM beamline at ESRF. Three experiments were carried out on two diffraction beamlines at the SOLEIL synchrotron, namely DiffAbs for experiments A and B and Cristal for experiment C. These first experiments with the prototype required the specific data treatment discussed here. The experiments are described below with setup differences according to the X-ray energy value, the sample-to-detector distance and the sample environment. Experiment A was dedicated to study of the elastic response of in situ deformed nanostructured metallic thin films, experiment B to the elastic-plastic deformation of a duplex steel, and experiment $\mathrm{C}$ to the growth of multi-walled carbon nanotubes. The reasons for using the XPAD3.1 detector were different for each experimental case, which in turn illustrates the performance and versatility of the detector.

The response of the XPAD3.1 detector varies with X-ray energy and with the geometrical setup, including the sampleto-detector distance. The specific design of the XPAD3.1 (description in \$2.1) induces some spatial distortions. Geometrical corrections must necessarily be applied on the data images as was also shown for another hybrid pixel detector (Hülsen et al., 2005). For the analysis of the diffraction images, it was also necessary to remove spurious signals and defective pixels or regions of no interest.

The goal of this article is to present a compilation of the calibration procedures developed to correct non-uniformities and distortions of data images of the prototype XPAD3.1 detector, as well as image cleaning procedures and algorithms for the intensity integration of the diffracted signals in the 
light of three experimental cases. Typical scientific results of the above-mentioned experiments are illustrated in the last part of this paper. More detailed analyses will be presented in articles from the different teams. The aim here is to give to a standard synchrotron user tools to analyse data acquired with XPAD-type detectors.

\section{Experimental setup}

\subsection{XPAD3.1 prototype detector}

The XPAD3.1 detector comprises eight modules of seven hybrid integrated circuits (chips) on a single silicon sensor (Medjoubi et al., 2010). A schematic description of the detector is given in Fig. 1. The silicon diode sensor has its rear face pixelized and each pixel is coupled via 'bump-bonding' to an electronic counting device in a dedicated circuit. Hybrid pixel detectors work in a single-photon-counting pixel mode.

A single chip contains $120 \times 80$ pixels, each of them measuring $130 \times 130 \mu \mathrm{m}$, except for the first and last columns of the chip, which have a nearly 2.5 times larger size in the horizontal direction. For the prototype version, the total size of the detection surface is $12 \times 7.5 \mathrm{~cm}$. Modules are assembled in tiles, which are inclined with an angle $\alpha$ of $7.5^{\circ}$ with respect to the vertical direction of the detector plane, with some superposition zones in order to minimize dead areas. Even in this case, some shadowed areas still exist (horizontal lines of a few pixels width), the width of the dead zones depending on the mounting accuracy of the modules and the geometry of the experiment. Dead lines between the modules should be taken into account to correct raw data images. According to the detector geometry, reliable image corrections can be carried out to obtain the correct diffraction angle corresponding to each pixel, as will be discussed in detail in the following sections.

(a)

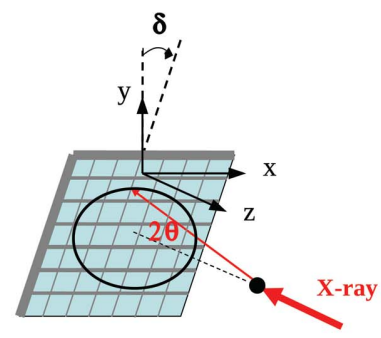

(b)

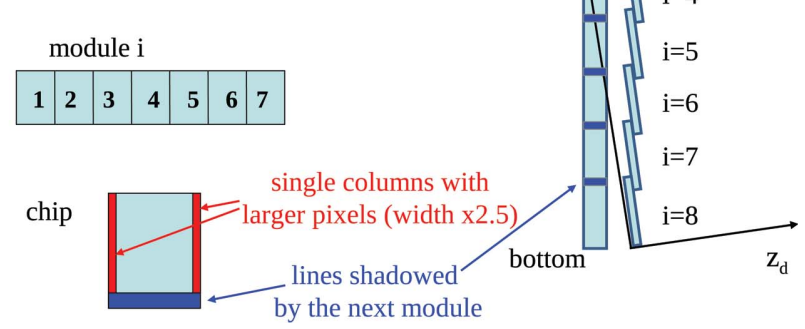

\section{Figure 1}

Schematic view of the geometry of the XPAD3.1 detector. (a) Experimental geometry with the tilt angle $\delta$ between the average surface of the detector and the vertical direction y. $2 \theta$ is the Bragg angle. (b) Description of a single module containing seven chips. (c) Side view of the detector showing the tiling of the modules with the tiling angle $\alpha$.
Table 1

The three experimental configurations using the XPAD3.1 detector.

The larger acquisition time for experiment $\mathrm{A}$ is partly owing to the use of the eight-bunch mode (corresponding to $80 \mathrm{~mA}$ in the storage ring) rather than the multi-bunch mode $(400 \mathrm{~mA})$ which is used for experiments B and C.

\begin{tabular}{lclll}
\hline Experiment & $E(\mathrm{keV})$ & $D(\mathrm{~mm})$ & $\begin{array}{l}\text { Acquisition time } \\
\text { per image }(\mathrm{s})\end{array}$ & $\begin{array}{l}\text { Beam size }(\mathrm{HWHM}) \\
\left(\mathrm{H} \times \mathrm{V} \mathrm{mm}^{2}\right)\end{array}$ \\
\hline A & 8.8 & 536 & 30 & $0.32 \times 0.37$ \\
B & 10.7 & 860 & 5 & $1.0 \times 0.3$ \\
C & 17.1 & 152 & 1 & $0.4 \times 0.1$ \\
\hline
\end{tabular}

For all three experiments, the detector was mounted on the $\delta$ arm (corresponding to rotation in the vertical scattering plane) of a six-circle $\kappa$ goniometer. The direct X-ray beam impinged perpendicularly on the detector surface $(\delta=0)$ in experiment $\mathrm{C}$, whereas the detector was positioned at nonzero $\delta$ values in experiments $\mathrm{A}$ and $\mathrm{B}$. The $\delta$ angle can also be described as the tilt of the detector with respect to the vertical direction (see Fig. 1). The energy, sample-to-detector distance $D$, acquisition time and $\mathrm{X}$-ray beam size (horizontal $\times$ vertical) are reported in Table 1 for each of the three experiments.

\subsection{Description of experiments}

The scientific background for each experiment is briefly introduced to illustrate the fact that the detector can be useful in a range of different fields.

The first experiment, referred to in the text as experiment A, concerns the mechanical characterization of metallic thin films deposited on a compliant substrate using in situ biaxial tensile tests coupled to synchrotron X-ray diffraction (XRD). The mechanical properties of thin crystalline films are investigated with respect to their microstructure and often depart from their bulk counterparts (Geandier et al., 2009; Faurie et al., 2009, 2010). Thin films can exhibit high residual stress states, strong texture components, a high density of defects, small grain size and a high ratio of interfaces. X-ray diffraction is well suited to the measurement of small elastic strains with high accuracy and the examination of strain partitioning between different crystallographic phases and/or different texture components. The samples investigated are $\mathrm{W} / \mathrm{Cu}$ nanocomposite thin films deposited on polyimide cruciform substrates (Geandier et al., 2010; Djaziri et al., 2010). The small diffraction volume (film thickness of $200 \mathrm{~nm}$ ) combined with the nanometric size of the grains yields very broad and lowintensity diffraction peaks. Improving the data statistics by adopting a long exposure time may imply material creep and substrate relaxation, leading to inaccurate measurements. Hence the main reasons for using the XPAD3.1 detector for this experiment were its fast acquisition time and its twodimensional geometry, taking advantage also of the intense synchrotron radiation. Data acquired using area detectors can be reduced to $2 \theta$ line scans by performing data integration along the azimuthal direction, allowing one to improve statistics. 
A specially developed biaxial tensile device (Geandier et al., 2010) was installed on the DiffAbs beamline goniometer. In the $\mathrm{W} / \mathrm{Cu}$ multilayered thin films, composed of 60 periods of $4 \mathrm{~nm}(3 \mathrm{~nm}$ of $\mathrm{W}$ and $1 \mathrm{~nm}$ of $\mathrm{Cu})$, we focused on the mechanical response of the tungsten phase. The X-ray energy was set at $8.8 \mathrm{keV}$, slightly above the $\mathrm{Cu} K$-absorption edge, to avoid copper fluorescence and thus a complex data treatment based on fluorescence filtering. Diffraction patterns were recorded on the XPAD3.1 detector placed on the $\delta$ arm $530 \mathrm{~mm}$ away from the specimen in order to avoid any collision during measurements. The $2 \theta$ angular aperture of a pixel is about $0.014^{\circ}$. It is worth noting that the elastic strain of interest results in a small Bragg peak shift of about $0.01^{\circ}$ (0.7 pixels), corresponding to $1 / 200$ of the peak width (full width at half-maximum $\simeq 2^{\circ}$ ). Data images (Fig. $2 a$ ) were obtained during a step-by-step deformation procedure. X-ray measurements were performed for different sample orientations for a given level of applied load. Seventeen different inclination angles for two different azimuth angles (corresponding to the two loading directions of the cruciformshaped sample) could be used to measure the Bragg peak shifts. An integration procedure (see $\$ 3.3$ ) was used to get a classical diffractogram from which the Bragg peak position is obtained using a fitting procedure (Pearson VII function and linear background). The lattice strain is thus measured with a very high accuracy (about $5 \times 10^{-5}$ ) thanks to the so-called $\sin ^{2} \psi$ analysis of the 17 data points (Dölle, 1979; Djaziri et al., 2011; Hauk, 1997).

Experiment $\mathrm{B}$ is dedicated to the investigation of the mechanical response of a duplex steel specimen containing two phases, $\sim 50 \%$ body-centred cubic $(\alpha)$ ferrite and $\sim 50 \%$ face-centred cubic $(\gamma)$ austenite. Special attention is paid to the stress and strain intragranular heterogeneities at small

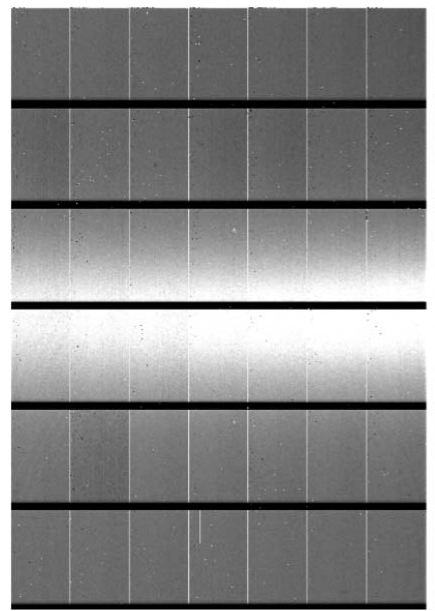

(a)

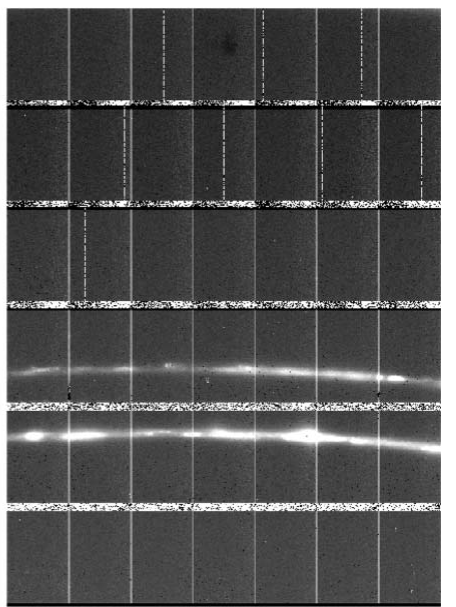

(b)
Figure 2

Typical raw images obtained on the XPAD3.1 detector, showing small parts of Debye-Scherrer rings for $(a)$ experiment A (intensity range 0 $1800)$ and $(b)$ experiment $B$ (intensity range $0-140000$ ). The images are plotted in log scale. Only modules $2-7$, centred on the scattered signals, are shown. The dramatic difference in peak width results from the different microstructures (nanometre versus micrometre grain sizes). Some dead pixels and dead lines can be observed on both images. strain, in particular during the elastic-plastic transition. Diffraction-based techniques are ideally suited for such studies since they allow the characterization of the average elastic strains (shift of Bragg peaks) and the strain heterogeneities (peak broadening) (Letouzé et al., 2002; Le Bourlot, 2012). The intrinsic low noise, high dynamic range, large detection area and short readout time of the XPAD3.1 detector were clearly an advantage. In particular, the possibility of filtering the X-ray fluorescence scattering of iron enhanced the signal-to-noise ratio. More generally, rapid and accurate acquisitions open the possibilities to map the orientation dependence of the internal stress within the material, which is particularly interesting for material deformation studies.

A $5000 \mathrm{~N}$ DEBEN tensile rig was installed on the $x y z$ translation tables of the six-circle goniometer. Diffraction signals originating from various $\{h k l\}$ lattice planes were recorded on the detector placed on the $\delta$ arm at $860 \mathrm{~mm}$ from the specimen (Fig. $2 b$ ). The energy was set at $10.7 \mathrm{keV}$, i.e. small enough to reach an adequate $2 \theta$ resolution but large enough for the fluorescence signal of the Fe edge $(7.11 \mathrm{keV})$ to be separated and removed by the XPAD electronics. Data images were obtained during a continuous sample straining. The sample orientation was kept fixed during the experiment, and scattered signal originating from two families of diffracting planes, $\alpha\{211\}$ and $\gamma\{220\}$, could be measured simultaneously on the detector. Diffraction patterns were recorded continuously, i.e. every $\sim 5 \mathrm{~s}$. Since only the relative displacement and broadening of Bragg peaks (between two successive loading steps) was of interest, the data are not sensitive to parallax errors (Hülsen et al., 2005). Considering the signal-to-noise ratio of the data the maximum intensity of the Bragg peaks, the photon noise based on Poisson statistics, and the variances of the first and second moments of the Bragg peaks (providing the uncertainties for peak position and peak width) could be estimated (Le Bourlot, 2012). We could reach a strain resolution of $10^{-4}$, corresponding to a shift or broadening of the Bragg peaks as small as 0.2 pixels. This setup allows a precise investigation of lattice strain evolution during the elasto-plastic transition.

Experiment $\mathrm{C}$ is an in situ time-resolved XRD study of the growth mechanism of multi-walled carbon nanotubes (CNTs) synthesized by aerosol-assisted catalytic chemical vapour deposition (AA-CCVD) through simultaneous injection of both carbon and iron-based catalytic precursors (Pinault et al., 2005). A specific reactor and furnace have been developed to perform such experiments on XRD synchrotron beamlines. Time-resolved study of the CNT growth and in particular their nucleation required short acquisition times. Moreover, the measurement of very weak scattering signals from the growing CNTs and the iron-based catalyst nanoparticles took advantage of the high sensitivity and of the low noise of the detector. XRD patterns were recorded as a function of time during nucleation and growth of the CNTs. An acquisition time of $1 \mathrm{~s}$ was chosen in order to detect the weak scattering signal from the small quantity of matter under investigation. Such an acquisition time is rather long with respect to the nominal 
readout time of the detector of $2 \mathrm{~ms}$ but it was necessary owing to the rather high energy value chosen $(17.1 \mathrm{keV})$, at which the sensitivity of the detector decreases (Medjoubi et al., 2010). The small sample-to-detector distance and the high energy were intended to give access to a large $Q$ wavevector domain. Parallax effects implying a peak position shift (Hülsen et al., 2005) were not observed because the scattered signals are broad ( $\sim 15$ pixels). In this experiment, where the sample-to-detector distance is small $(152 \mathrm{~mm})$, the geometrical corrections described in $\$ 3.2$ were the most needed in comparison with experiments $\mathrm{A}$ and $\mathrm{B}$.

\section{Image processing}

The corrections and algorithm for intensity integration were programmed in ImageJ macro language (http://rsbweb.nih. gov/ij/) and in Python scripts. Batches of data are submitted to the same image processing algorithms described below.

\subsection{Image cleaning}

Data processing requires the specification of the direct beam position and the angular aperture of a pixel. For this purpose, the XPAD3.1 detector was placed in the direct beam using filters to prevent burn-out of the detector. An image is captured and the direct beam coordinates are determined. Then, the detector is shifted by some degrees in angle $\delta$ in order to obtain the angular aperture of a pixel.

3.1.1. Grid correction. Owing to the particular stacking of XPAD3.1 modules (tiling design), some of the pixels lines are hidden by the neigbouring module (see Fig. 1). This induces a pixel shift between the modules. To correct this image distortion, we have used a mask made of a metallic plate with an array of well defined holes placed in front of the detector illuminated by the X-ray scattered signal from a polyimide foil, the detector being placed far away (about $1 \mathrm{~m}$ ) to ensure an illumination of its surface with an almost flat and uniform intensity X-ray signal. An image of this mask is captured, and each module shift is then calculated to recover a constant distance between the illuminated grid holes. These shifts are taken into account for creating a new corrected image of the detector.

As mentioned above, the chips composing the modules have approximately 2.5 times larger pixels at their edges. These particular pixels are replaced by $\sim 2.5$ pixels of the same size as the others, renormalizing the measured intensities by a factor $\sim 1 / 2.5$. Taking account of all these corrections, a new corrected image is generated.

3.1.2. Detection of dead pixels. The detection of dead, inefficient or unreliable pixels is of utmost importance for obtaining clean data of high accuracy. For this purpose, raw images were tested against a number of filters, and pixels that did not pass all tests were removed. First, a binary mask indicating the position of evident bad pixels was constructed during the process. Then, the intensity of each pixel in the data image was systematically compared with the average intensity of neighbouring pixels. If this difference is larger than a given threshold, then the pixel might not be faithful. The threshold was adjusted by a trial-and-errors procedure on a series of typical detector images so that all bad pixels that could be detected manually were detected by the algorithm. A pixel was then skipped if its intensity was 20 times larger than the mean of its neighbours or smaller by $1 / 20$ than the minimum value of its neighbours. Doing so, we probably eliminated more pixels than necessary, but this solution was found to be satisfactory for experiments $\mathrm{A}$ and $\mathrm{B}$, as the maximum intensity of Bragg peaks after azimuthal integration (see $\$ 3.3$ ) is large enough (typically larger than $10^{4}$ ) to give accurate results.

3.1.3. Detection of inefficient chips. The low X-ray energy used in experiments A and B is close to the limit of capability of the XPAD3.1 prototype (the detector calibration on setting an energy threshold close to the noise level had not been perfectly mastered at that time, and consequently some noise was induced by the power supply). On some chips, a global drift of the intensity could be randomly observed. For instance, about 8 out of 34 images are affected in experiment A during the $20 \mathrm{~min}$ acquisition procedure, i.e. for a given loading state. With the actual setup geometry (detector far away from the sample in experiments A and B), the average background intensity measured on each chip should not deviate much from that of the adjacent chip (left or right). An illustration with data is provided in Fig. 3, where chip 6 clearly exhibits a peculiar behaviour due to a difficult detector calibration at the energy values in experiments $\mathrm{A}$ and $\mathrm{B}$. We systematically checked the module average intensity and compared it with the most different chip average intensity. This chip was then skipped when its intensity deviated by more than $9 \%$ from the module average; the algorithm looped as long as a deviant chip was found. This analysis is particularly sensitive for experiment A since a slight change of the integrated intensity due to the unreliable chip may induce a significant change in the Bragg peak position compared to the
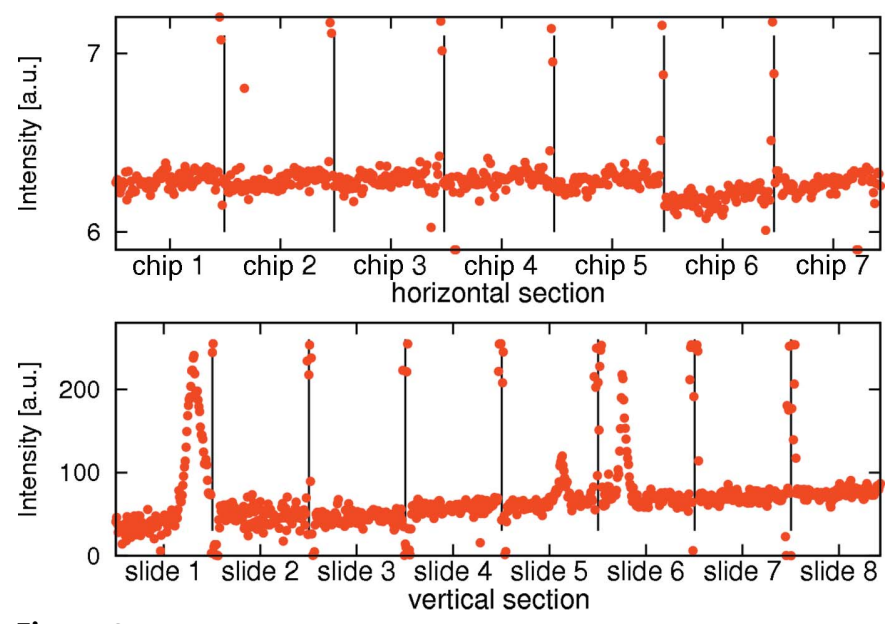

Figure 3

Examples of horizontal (top) and vertical (bottom) sections of the raw XPAD image (data from experiment B). Pixel lines separating chips (top) and modules (bottom) are clearly visible. The overall gradient of the intensity over a single chip (top) or module (bottom) is shown and is corrected with the flat-field correction. 
required resolution. Fig. 4 clearly illustrates the results of $\mathrm{X}$-ray strain measurements and the absolute necessity of using the algorithm that removes the defective chips. Obviously, this procedure is not necessary if higher energies are used with the prototype XPAD3.1.

3.1.4. Flat-field and background corrections. Non-uniform response of the detector over the various pixels depends on the X-ray energy. The non-uniformity of the detector response was the most drastic for the lower-energy experiments (A and B): it showed up as an increase of the intensity from the left to the right of each chip. No flat-field correction was necessary for experiment $\mathrm{C}$. The 'left-right' effect was due to an unbalanced power distribution in the chip, which has now been corrected. Calibration of the detector was performed at the working X-ray energy $\left(E_{\text {eff }}\right)$ by setting a discriminator threshold at $E_{\text {eff }} / 2$ for the whole chip with fine adjustment for each pixel electronic device. The detector could not be calibrated accurately at the limit of its efficiency $E_{\text {eff }} \simeq 8 \mathrm{keV}$ (no more chip response at energies smaller than $\sim 6 \mathrm{keV}$ ).

Flat-field correction is performed by dividing the images with a reference image of uniform intensity. This can be obtained when the detector is illuminated using a fluorescence signal from a point source placed far away. For experiment A, 30 flat-field patterns recorded for $600 \mathrm{~s}$ each were generated from the X-ray fluorescence signal of a copper sheet illuminated by a $9 \mathrm{keV} \mathrm{X}$-ray beam (above the copper absorption threshold). For experiment B, the flat field was obtained similarly from the X-ray fluorescence of a GeO powder. Single flat-field images were then summed up into a final flat-field image.

Flat-field images were further used for data cleaning, i.e. removing all pixels having intensity deviating by more than $20 \%$ from the average image intensity. A mask of 'dead' pixels was calculated only once and then used for the processing of all data images. Let us also stress that, for XPAD3.1, dark-field

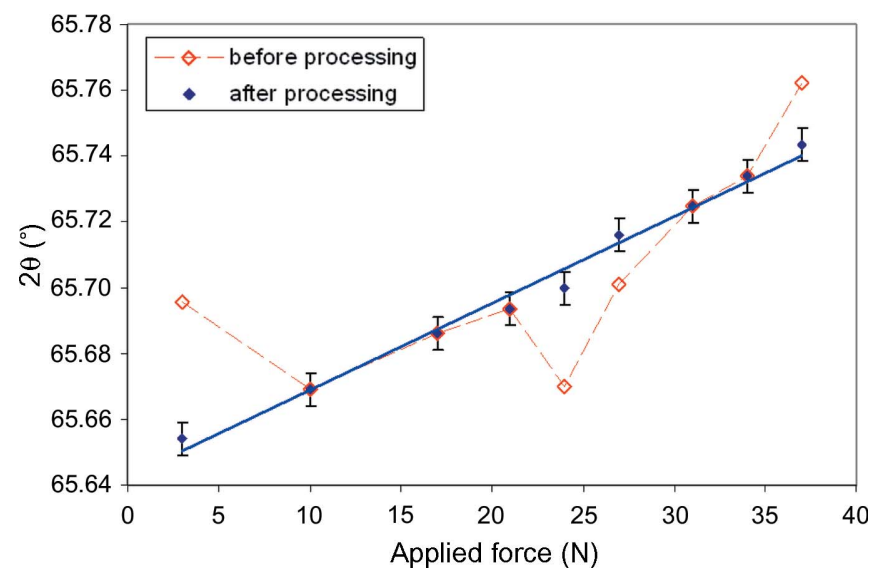

Figure 4

Bragg peak position ( $2 \theta$ angle) as a function of the applied load on the composite film substrate. Red open symbols are the data before image processing by the 'image cleaning' algorithm. Dark blue filled points are the data obtained after image processing. The solid blue line is the linear fit of this data as should be the case in the elastic linear regime. It is noteworthy that the high probability of having defective chips with XPAD3.1 is induced by the low energy used in experiment $A$. Uncertainties are estimated to be $\Delta 2 \theta= \pm 0.005^{\circ}$. correction is not necessary since the detector exhibits no noise; recorded dark-field images (with typical acquisition times of 1-10 s) are found with zero intensity for all not-dead pixels.

An illustration of the flat-field and background corrections is provided in Fig. 5. It can be seen in particular that the overall intensity gradients appearing on each chip on the raw images (bottom-top and left-right gradients shown in Fig. 3) are nicely corrected in the final image. An affine background is then subtracted for later peak analysis after azimuthal integration.

\subsection{Geometrical corrections}

The tiling geometry of the modules characterized by the tiling angle $\alpha$, and the average tilt angle or vertical angle $\delta$ (see Fig. 1), have a direct consequence on the distortion of the XPAD3.1 images. The intersection between the diffraction cone and the plane of the detector surface departs from the circular shape (He, 2009) as soon as the detector surface is no longer perpendicular to the direct X-ray beam, resulting in an
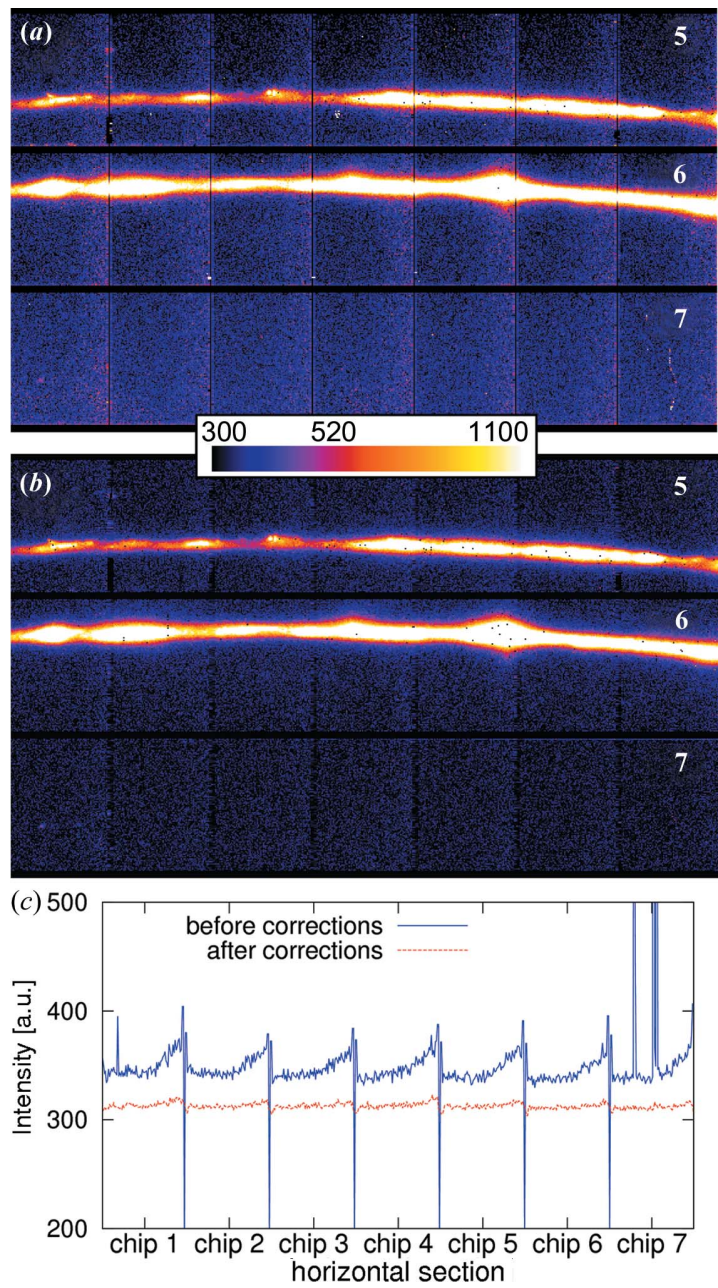

Figure 5

Image processing applied to a data image (log scale) from experiment B (only modules 5-7 are shown): (a) image after the grid correction procedure; $(b)$ image after the cleaning and flat-field correction procedures; $(c)$ average intensity over module 7 before (blue line) and after (red line) the grid, cleaning and flat-field corrections. 
elliptical shape. In the particular case of this detector composed of tiled modules, arcs of ellipses, which are not continuously interconnected, will be observed from one module to the other.

Let us detail here the analytical expressions we used for taking into account these effects. The pixel coordinates are $\left(x_{\mathrm{d}}\right.$, $\left.y_{\mathrm{d}}, z_{\mathrm{d}}\right)$ in the frame attached to the detector, with axes $x_{\mathrm{d}}$ and $y_{\mathrm{d}}$ parallel to the module planes and $z_{\mathrm{d}}$ perpendicular to them (see Fig. 6). The origin $\left(O_{N}\right)$ of this frame is located at the lowest corner of the bottom module $(N=8)$. The laboratory reference frame $(x, y, z)$ has its origin $O$ located at the sample position, corresponding to the vertex of the diffraction cone. The direct X-ray beam is along the $z$-axis direction.

In the laboratory reference frame, the cone of diffraction is defined by its half-apex angle $2 \theta$. The Bragg angle $\theta$ is given by

$$
\theta=\frac{1}{2} \arctan \left[\left(x^{2}+y^{2}\right)^{1 / 2} /|z|\right] \text {. }
$$

To obtain the corresponding relation from pixel positions on the detector image, coordinates $(x, y, z)$ should be expressed in terms of the detector coordinates $\left(x_{\mathrm{d}}, y_{\mathrm{d}}, z_{\mathrm{d}}\right)$ :

$$
\begin{aligned}
& x=x_{\mathrm{d}}, \\
& y=y_{O_{N}}+y_{\mathrm{d}} \cos (\delta-\alpha)-z_{\mathrm{d}} \sin (\delta-\alpha), \\
& z=z_{O_{N}}+y_{\mathrm{d}} \sin (\delta-\alpha)+z_{\mathrm{d}} \cos (\delta-\alpha),
\end{aligned}
$$

with

$$
\begin{aligned}
& y_{O_{N}}=D \sin \delta-k l \cos \delta / \cos \alpha, \\
& z_{O_{N}}=-D \cos \delta-k l \sin \delta / \cos \alpha,
\end{aligned}
$$

and

$$
z_{\mathrm{d}}=(N-i) e=(N-i) l \tan \alpha
$$

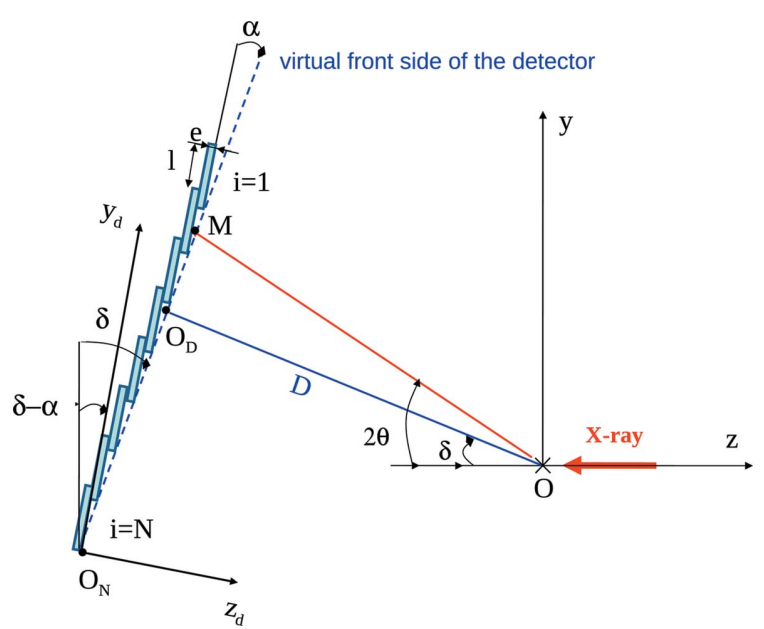

Figure 6

Schematic view of the XPAD3.1 detector in the detection geometry. The sample is placed at the origin $O$ of the laboratory frame $(x, y, z)$, corresponding to the vertex of the diffraction cone with $2 \theta$ Bragg angle. $D$ is the orthogonal distance between the sample and the virtual front side of the detector. $O_{D}$ is the intersection of the line perpendicular to the virtual front side with this side (i.e. $O_{D}$ corresponds to the direct beam for $\delta=0) . O_{N}$ is at the bottom corner of the lowest module in the $y z$ plane $\left(x=x_{d}=0\right)$. The detector frame $\left(x_{\mathrm{d}}, y_{\mathrm{d}}, z_{\mathrm{d}}\right)$ has its origin at $O_{N}$.
$D$ being the distance between the sample and the virtual (plane) front side of the detector, and $e$ and $l$ being, respectively, the thickness and the uncovered length of each module (see Fig. 6). The fractional number $k$ is defined by the segment $k=\left|O_{N} O_{D}\right| \cos \alpha / l ; k=N / 2$ if the direct beam is at the centre of the detector when $\delta=0$.

The geometrical corrections have been applied to data images for experiment C. As shown in Fig. 7(a) where corrections are not yet taken into account, the positions of the same 002 diffraction peak of the CNTs are found to be different in the horizontal and vertical directions because of the distortion of the diffraction rings (see inset of Fig. 7a). Therefore, geometrical corrections have been considered to reconstruct the diffraction pattern in Fig. 7(b), in which diffraction rings are now circular but where the geometry of the chips appears distorted instead of being rectangular. The geometrical corrections were performed by adjusting the value of $(\alpha-\delta)$ of equation (2) in order to fit the position of the 002 peak at its known value $Q=1.81 \AA^{-1}$ (Cambedouzou et al., 2012). The fitting procedure was also checked with a $\mathrm{CeO}_{2}$ powder calibration sample. The theoretical value given by the constructor of the detector is $\alpha=7.5^{\circ}$, and the uncertainty on $(\alpha-\delta)$ was estimated to be $0.03^{\circ}$ with a fitted value $\delta=0.6^{\circ}$. Let us recall that the origin $2 \theta=0$ was defined by the direct beam position procedure described above. The geometrical corrections algorithm described here gives the correct values of the diffraction angles [or the corresponding wavevector $Q=(4 \pi / \lambda) \sin \theta$ ] in the vertical and horizontal directions (see inset of Fig. $7 b$ ). Such geometrical corrections were much needed for the wide-angle scattering measurements of experiment $\mathrm{C}$ because of the short distance $D$ (see Table 1).

\subsection{Signal integration}

The geometrical corrections detailed previously allow us to connect the position of each pixel and the corresponding scattering angle $2 \theta$. Azimuthal integration of images is performed to obtain the average intensity distribution $I(2 \theta)$ of the Debye rings. This process consists in integrating the corrected images along the azimuth, for the azimuthal angular range available in the image. Two integration methods have been compared, as detailed below, depending on whether the intensity distribution on the detector image is interpolated at the subpixel scale or not.

The first integration trials were performed without subpixel interpolation. The $2 \theta$ range available in the detector image is discretized into several channels $2 \theta_{i}$. Then, for each pixel within the region of interest of the detector image, the corresponding $2 \theta$ value is computed and the pixel intensity is added to the nearest $2 \theta_{i}$ channel. Besides simplicity, this method has the advantage of not introducing any assumption about the shape of the intensity distribution between neighbouring pixels. Considering the fact that each pixel is attributed to the nearest channel, the number of channels should not be too important otherwise some channels would not exhibit a representative intensity level at the end of the inte- 
gration process. An optimal increment $\delta 2 \theta_{i}$ between adjacent channels is $4 / 3$ of the pixel size. To check the accuracy of the method, theoretical detector images have been generated and integrated with this procedure. We used for this purpose a rather difficult case, namely a sharp and narrow analytical Gaussian peak with an FWHM of only 3 pixels and an intensity ratio between neighbouring pixels of around 1/1000. As shown in Fig. 8, the spectrum integrated with this method does not really match the theoretical spectrum that can be calculated after analytical integration of the Gaussian Debye ring. Values of $\delta 2 \theta_{i}$ smaller than $4 / 3$ pixels enhance the noise of the integrated spectra, as there are not enough pixels to populate each channel. For larger increments, integrated spectra are artificially spread out. A significant shift of Bragg peak position is also apparent, depending on the chosen value for $\delta 2 \theta_{i}$. As anticipated, for the very sharp peaks of interest in experiment $\mathrm{B}$ (FWHM of $\sim 3$ pixels), this integration method is not accurate enough.



(a)
A more advanced integration procedure, used for experiment B, can be achieved by making use of subpixel interpolation of the image intensity, aiming at generating a new image with continuous intensity distribution from which the intensity can be easily integrated along any direction. The advantage of this method is that the integrated spectrum is no longer sensitive to any discretization step. However, subpixel interpolation has to be performed carefully to avoid the introduction of any artefacts in the original image. For this application, we used a bilinear interpolation which provides the intensity at any position according to the intensity of the four nearest pixels. If we choose a coordinate system in which these four nearest pixels have coordinates $(0,0),(0,1),(1,0)$ and $(1,1)$, then the interpolation formula simplifies to

$$
\begin{gathered}
I(x, y) \simeq I(0,0)(1-x)(1-y)+I(1,0) x(1-y) \\
+I(0,1)(1-x) y+I(1,1) x y, \\
\forall 0 \leq x, y \leq 1 .
\end{gathered}
$$
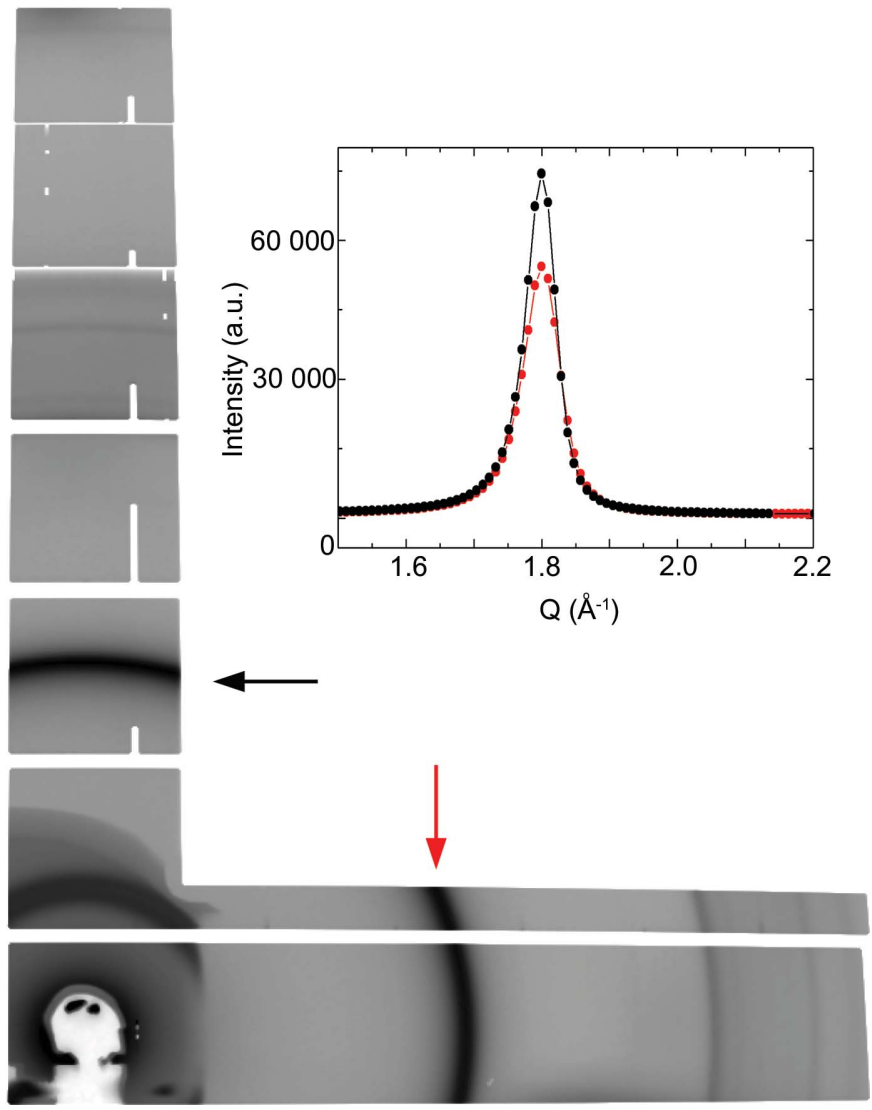

(b)

\section{Figure 7}

Illustration of the geometrical corrections applied to data from experiment $\mathrm{C}$. The available image area is determined by the L-shaped exit window of the furnace; the rest of the detector image is not shown (no detection). (a) Raw data with (002) CNT scattering peak at the end of the aerosol injection; (b) corrected image. Both images are shown in log scale. Note the slightly curved edges of the modules after the applied geometrical corrections. Insets: integrated intensity on horizontal and vertical branches of the L-shaped exit window [grey (red in the electronic version of the journal) and black dots] of the 002 scattering peaks indicated by grey (red) and black arrows in the horizontal and vertical directions. The uncertainty on the peak position is estimated to be $\Delta Q= \pm 0.01 \AA^{-1}$. 
Interpolation was disregarded as soon as one of these four neighbour pixels was declared dead. Contrary to the previous algorithm, subpixel interpolation requires, for each pixel, a procedure to determine nearest neighbours. This latter point is not trivial owing to the nonlinear geometry of the XPAD3.1, with discontinuities at module edges that can lead to possibly missing information. In experiment $\mathrm{B}$, we worked with only one module at a time as the interesting part of the Debye rings has been positioned in the middle of a module. This integration method has been checked against the analytical image introduced above (Fig. 8). It can be seen that the results are very satisfying. Not only is the peak position accurate but also its shape is preserved. The bilinear subpixel interpolation procedure leads to a shift of Bragg peaks (compared to their theoretical positions) corresponding to an elastic strain of $\sim 10^{-4}$, whereas shifts an order of magnitude larger are obtained with the first procedure described above. As for the peak width, interpolation and integration lead to a relative error of only a few percent. The bilinear interpolation seems thus well adapted even in the case of very narrow and sharp peaks, for which one might think that higher-order interpolation (e.g. bicubic or biquintic) would be required.

\section{Typical results}

Typical results are given in this section in order to illustrate the quality of the data obtained with the prototype XPAD3.1 detector. Deeper analysis and interpretations of the results will be presented in forthcoming articles from our different teams.

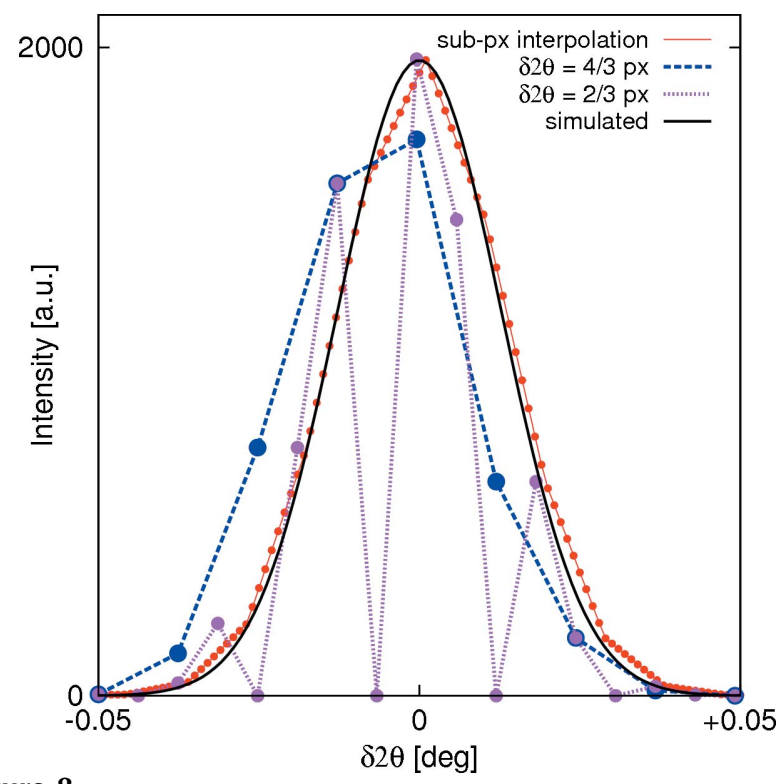

\section{Figure 8}

An example of Bragg peak intensity $I(2 \theta)$ obtained from an integration of a theoretical image simulated by ray tracing. The expected result is a Gaussian peak having an FWHM of 3 pixels. The integration is performed with or without subpixel interpolation, for an azimuthal integration over $2^{\circ}$ around the azimuth angle of $90^{\circ}$. When no subpixel interpolation was used, different values for the increment $\delta 2 \theta$ were investigated as a function of the pixel aperture $(\delta 2 \theta=2 / 3$ pixels and $\delta 2 \theta=4 / 3$ pixels $)$. (Shown in colour in the electronic version of the journal.)
Experiment A (Pprime team) focuses on the elastic characterization of metallic thin films under biaxial tensile tests. Elastic strains were obtained by studying the diffraction peak position shift for each loading state. Thus, the main focus of interest for experiment $\mathrm{A}$ is to obtain diffraction peak positions with an accuracy of at least $10^{-4}$, since the applied strains are very small and consequently the peak position shift is also small. As shown in Fig. 4, the peak position shift is extracted accurately in spite of its significant width (owing to nanocrystalline coherent volume) and is observed to vary linearly with the applied load since the investigated load range remains within the elastic domains of both the polyimide supporting substrate and the film. The analysis method described in this paper is an easy way to improve results.

Data analysis from experiment B (LSPM and PIMM teams) is presented in Fig. 9. A proper micromechanical interpretation of diffraction data requires the calculation of the moments of Bragg peaks $I(2 \theta)$ (Bretheau \& Castelnau, 2006). The first moment of $I(2 \theta)$ defines the peak position (or more accurately the position of its centre of mass) and allows estimation of the average elastic strain in the diffracting volume along a direction parallel to the diffraction vector $\mathbf{Q}$. The second-order moment of $I(2 \theta)$ provides indications about the peak width; it is associated with the second moment of this elastic strain. From these two moments, the standard deviation of elastic strain in the diffracting volume, which characterizes the strain heterogeneity inside the specimen, can be easily computed (Le Bourlot, 2012). Here, the heterogeneity of elastic strain is due to (i) the elaboration process of the material (hot rolling) and (ii) the external loading applied in situ. It is a consequence of the mechanical interactions arising between grains due to their anisotropic elastic and plastic behaviours, the different thermal expansion coefficients between $\alpha$ and $\gamma$ phases, and lattice distortions due to the dislocations' structure.

As shown in Fig. 9, it can be checked that shifts of $\alpha\{211\}$ and $\gamma\{220\}$ peaks remain perfectly proportional along the two

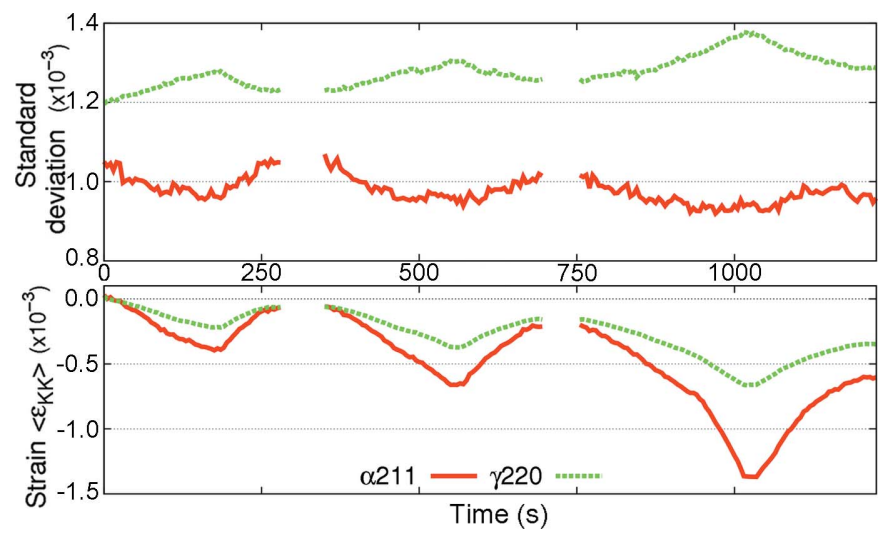

Figure 9

Bottom: elastic (lattice) strain corresponding to the shifts of the $\alpha\{211\}$ and $\gamma\{220\}$ peaks along the three loading-unloading cycles. Top: corresponding strain heterogeneities in the diffracting volume associated with peak broadening. The specimen response is indicated with respect to the time required for in situ loading. (Shown in colour in the electronic version of the journal.) 


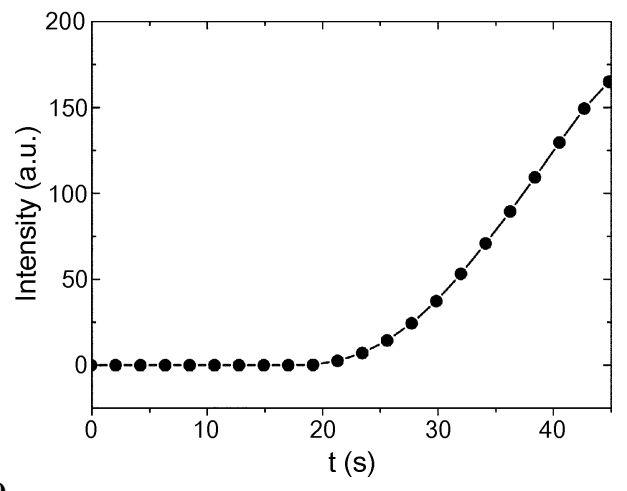

Figure 10

Evolution of the integrated intensity of the 002 scattering peak of a CNT as a function of time. The increase of intensity corresponds to the CNT growth. The injection of the precursors starts at time $t=0$.

first loading-unloading cycles shown in Fig. 9, in agreement with a pure elastic mechanical response of the specimen. Peak $\gamma\{220\}$ also exhibits smaller lattice strain compared to $\alpha\{211\}$, although Young's modulus of isotropic austenite polycrystals for the $\{220\}$ planes is about half that for the $\{211\}$ planes in ferrite. The peak shift can be explained when considering the mechanical interaction between $\alpha$ and $\gamma$ grains, e.g. with the help of micromechanical models (Letouzé et al., 2002; Faurie et al., 2009). The actual results are in good quantitative agreement with predictions of the thermoelastic self-consistent model (Le Bourlot, 2012).

As for the interpretation of peak widths, the data show a clear evolution of strain heterogeneity for the $\gamma\{220\}$ planes. The elastic strain heterogeneities increase linearly with the applied stress, as expected for linear elasticity. As clearly observed in Fig. 9, the corresponding data for $\alpha\{211\}$ are much more noisy. This is associated with the poorer smoothness of the original diffraction rings: they appear much more spotty for $\alpha\{211\}$ than for $\gamma\{220\}$ data, indicating smaller intragranular misorientations in $\alpha$ grains than in $\gamma$ grains. The coupling between the residual strain heterogeneity due to the elaboration process and strain heterogeneity due the in situ mechanical loading (associated with the elastic anisotropy of grains) might explain the decreasing line width for $\alpha\{211\}$. It should, however, be noted that these results characterizing intragranular strain heterogeneities (line width evolution) for elastically deformed polycrystals are to the best of our knowledge the first of the kind.

The objective of experiment C (LPS and LFP teams) was to study the mechanisms for CNT nucleation, growth and alignment. Nanotube growth occurs at $1123 \mathrm{~K}$ (Pinault et al., 2005) during the injection of aerosol (the conditions are described in detail elsewhere; Landois et al., 2011). As shown in Fig. 7, we were able to observe the 002 scattering peak at $Q=1.81 \AA^{-1}$ at $1123 \mathrm{~K}$, which is characteristic of the multiwalled carbon nanotubes [its position corresponds to the interwall distance in nanotubes (Pichot et al., 2004)]. Timeresolved XRD with XPAD3.1 allows us to follow every $2 \mathrm{~s}$ the evolution of the integrated intensity of the 002 peak related to the CNT growth (Landois et al., 2011). From the start of the injection, one could observe the appearance of the 002 peak after a delay of $20 \mathrm{~s}$, corresponding to an induction time before CNT growth (see Fig. 10). The increase of the intensity of the 002 peak corresponds to the growth of the multi-walled CNTs. This experiment was possible thanks to the XPAD3.1 detector in terms of (i) low noise in order to measure the very weak scattering from the small quantity of matter at the beginning of the CNT nucleation and (ii) fast readout time enabling time-resolved measurements. To the best of our knowledge, although in situ XRD studies of CNT growth have already been reported in the literature, the present experiment is the first time-resolved in situ study. It will thus bring new results concerning open questions in the field, such as the nature of the catalyst particles (Landois et al., 2011).

\section{Conclusion}

Calibration and correction procedures have been applied for data images obtained with the prototype XPAD3.1 hybrid pixel detector. We have detailed our procedures for corrections of XPAD3.1 images and for the signal integration in the context of three different diffraction experiments encompassing various X-ray energies, sample-to-detector distances and signal contrasts. The data obtained have fully taken advantage of the performance of the detector, namely its low noise and high dynamic range in detected signals and its short readout time. The present paper demonstrates that XPAD3.1 is a very promising detector for synchrotron diffraction measurements in materials science.

The analysis methods described in this paper are available as a plugin for the image processing software ImageJ, or as a Python procedure. Associated code can be made freely available to the community upon request to the authors.

We acknowledge the support of the DiffAbs and Cristal beamline teams for performing the experiments described in this paper and discussion with J. F. Bérar (D2AM, ESRF) about the correction procedures. Acknowledgements are made to the French National Agency of Research (ALUCINAN project) and RTRA 'Triangle de la Physique' for funding the project related to experiment $\mathrm{C}$.

\section{References}

Basolo, S., Bérar, J. F., Boudet, N., Breugnon, P., Chantepie, B., Clemens, J. C., Delpierre, P., Dinkespiler, B., Hustache, S., Medjoubi, K., Menouni, M., Morel, C., Pangaud, P. \& Vigeolas, E. (2008). Nucl. Instrum. Methods Phys. Res. Sect. A, 589, 268-274.

Bretheau, T. \& Castelnau, O. (2006). Rayons X et Matière (RX2006), ch. 5, pp. 123-154, edited by R. Guinebretière \& Ph. Goudeau. London: Hermès Science Publishing.

Cambedouzou, J., Landois, P., Rouzière, S., Pinault, M., Mocuta, C., Hennet, L., Thiaudière, D., Mayne-L'Hermite, M. \& Launois, P. (2012). In preparation.

Djaziri, S., Renault, P.-O., Hild, F., Le Bourhis, E., Goudeau, P., Thiaudière, D. \& Faurie, D. (2011). J. Appl. Cryst. 44, 1071-1079.

Djaziri, S., Thiaudière, D., Geandier, G., Renault, P. O., Le Bourhis, E., Goudeau, Ph., Castelnau, O. \& Faurie, D. (2010). Surf. Coat. Technol. 205, 1420-1425.

Dölle, H. (1979). J. Appl. Cryst. 12, 489-501.

Faurie, D., Castelnau, O., Brenner, R., Renault, P.-O., Le Bourhis, E. \& Goudeau, Ph. (2009). J. Appl. Cryst. 42, 1073-1084. 
Faurie, D., Djemia, P., Le Bourhis, E., Renault, P. O., Roussigné, Y., Chérif, S. M., Brenner, R., Castelnau, O., Patriarche, G. \& Goudeau, P. (2010). Acta Mater. 58, 4998-5008.

Geandier, G., Gélébard, L., Castelnau, O., Le Bourhis, E., Renault, P.-O., Goudeau, Ph. \& Thiaudière, D. (2009). IUTAM Symposium on Modelling Nanomaterials and Nanosystems, IUTAM Book Series Vol. 13, edited by R. Pyrz \& J. C. Rauhe, pp. 99-108. Dordrecht: Springer.

Geandier, G., Thiaudière, D., Randriamazaoro, R. N., Chiron, R., Djaziri, S., Lamongie, B., Diot, Y., Le Bourhis, E., Renault, P. O., Goudeau, P., Bouaffad, A., Castelnau, O., Faurie, D. \& Hild, F. (2010). Rev. Sci. Instrum. 81, 103903.

Hauk, V. (1997). Structural and Residual Stress Analysis by Nondestructive Methods: Evaluation, Application, Assessment. Amsterdam: Elsevier.

He, B. B. (2009). Two-Dimensional X-ray Diffraction. Hoboken: John Wiley and Sons.

Henrich, B., Bergamaschi, A., Brönnimann, C., Dinapoli, R., Eikenberry, E. F., Johnson, I., Kobas, M., Kraft, P., Mozzanica, A. \& Schmitt, B. (2009). Nucl. Instrum. Methods Phys. Res. Sect. A, 607, 247-249.
Hülsen, G., Brönniman, Ch. \& Eikenberry, E. F. (2005). Nucl. Instrum. Methods Phys. Res. Sect. A, 548, 540-554.

Landois, P., Rouzière, S., Pinault, M., Porterat, D., Mocuta, C., Elkaim, E., Mayne-L'Hermite, M. \& Launois, P. (2011). Phys. Status Solidi, 248, 2449-2453.

Le Bourlot, C. (2012). PhD thesis, Université Paris Nord, France.

Letouzé, N., Brenner, R., Castelnau, O., Béchade, J. L. \& Mathon, M. H. (2002). Scr. Mater. 47, 595-599.

Medjoubi, K., Bucaille, T., Hustache, S., Bérar, J.-F., Boudet, N., Clemens, J.-C., Delpierre, P. \& Dinkespiler, B. (2010). J. Synchrotron Rad. 17, 486-495.

Pichot, V., Launois, P., Pinault, M., Mayne-L'Hermite, M. \& Reynaud, C. (2004). Appl. Phys. Lett. 85, 473-475.

Pinault, M., Pichot, V., Khodja, H., Launois, P., Reynaud, C. \& Mayne-L'Hermite, M. (2005). Nano Lett. 5, 2394-2398.

Ponchut, C., Clément, J., Rigal, J. M., Papillon, E., Vallerga, J., LaMarra, D. \& Mikulec, B. (2007). Nucl. Instrum. Methods Phys. Res. Sect. A, 576, 109-112.

Teyssier, C., Bouchami, J., Dallaire, F., Idarraga, J., Leroy, C., Pospisil, S., Solc, J., Scallon, O. \& Vykydal, Z. (2011). Nucl. Instrum. Methods Phys. Res. Sect. A, 650, 92-97. 